\title{
British Psychophysiology Society Annual Meeting (2005): análise da produção
}

Geraldina Witter

Doutora em ciências, livre-docente em psicologia escolar. E-mail: gwitter@uol.com.br

Jamili Salem Souza

Mestre em Psicologia da Saúde pela Universidade Metodista de São Paulo - Umesp

E-mail: jamilirasoul@uol.com.br

\section{Resumo}

O objetivo foi analisar a produção científica $(N=85)$ veiculada durante o British Psychophysiology Society Annual Meeting, realizado em 2005 em Birmingham, na Inglaterra, considerando autoria, tipo de trabalho, característica do título, países participantes, instituições de origem dos pesquisadores, temática e estrutura geral do resumo. Os resultados mostraram predomínio da autoria múltipla (73\%) de trabalhos relatando dados de pesquisa (98\%). Não houve diferenças significantes quanto ao número de vocábulos usados no título até 9 (33\%) de 10 a 12 (29\%) e mais de 13 (37\%). Os países que mais apresentaram trabalhos foram Inglaterra (55,4\%), Alemanha $(10,7 \%)$ e Estados Unidos (10,4\%), sendo a Aston University a de maior presença ( $N=$ 12). Na temática houve grande dispersão, sendo mais freqüentes os estudos sobre funcionamento do cérebro e sobre os órgãos do sentido. Apenas seis resumos (7\%) usaram parágrafo. Os indicadores cientométricos permitem considerar que se trata de área que vem se desenvolvendo em consonância com os parâmetros da ciência.

\section{Palavras-chave}

Eventos científicos. Cientometria. Informação científica

British Psychophysiology Society Annual Meeting (2005): Productions analysis

\begin{abstract}
The aim was to analyze the scientific production $(N=85)$ circulated during the British Psychophysiology Society Annual Meeting, which took place in 2005 at Biaminghan, England, concerning authorship, type of work, characteristics of titles, participant countries, institutions of origin of research, thematic and general structure of the abstracts. The results showed predomination of multiple authorship (73\%). The countries that presented more papers were: England (55,4\%), Germany (10,7\%) and USA $(10,4 \%)$. Aston University presented more works $(N=12)$. There was a great variety of themes, but cerebral function and perceptual senses were the most recurrent ones. Only six (6\%) of the abstracts has used paragraph. According to scientific indicators, this area has been developed within science parameters.
\end{abstract}

\section{Keywords}

Scientific events. Scientometry. Scientific information

\author{
INTRODUÇÃO
}

A produção científica do conhecimento vem crescendo ao longo dos séculos mas é especialmente no século $\mathrm{XX}$ que ela se expande em ritmo espetacular e com produtos que mudaram substantivamente todos os aspectos da vida humana. O quadro resultante permite considerar que o século passado pode ser denominado o século da ciência (WITTER, 1994). Estes avanços continuam, em algumas ciências mais rapidamente do que em outras, no presente século, a despeito de perspectivas divergentes em alguns aspectos. Há consenso em vários aspectos como no reconhecimento de que a explosão de conhecimento requer avaliações sistemáticas, pois se, enriquecem e tornam mais eficiente a ciência, o produtor, o profissional, em cotrapartida sem critérios claros, sem evidências, sem criticidade e organização, as pessoas podem se perder no emaranhado de informações por vezes contraditórias, sem realidade, sem potencial de generalização e uso. (BERTERO, CALDAS, WOOD JR., 2005, ALTMAN, 1998).

Entre as múltiplas perspectivas envolvendo esta produção, duas são aqui enfocadas: a necessidade de tornar público o conhecimento alcançado e a de avaliar o produzido. Tornar público implica apresentar o trabalho para a comunidade científica e para a sociedade de um modo geral. Para dar a conhecer o produto entre os pares, espera-se que o trabalho seja submetido a eventos e publicado sde alguma forma (artigo, capítulo, livro). Para alcançar o grande público, deve ser reapresentado em nível compatível em periódicos de divulgação científica, artigos de jornais, folders e outras formas assimiláveis pelo cidadão não integrante do quadro de cientistas da área de saber que o gerou. Avaliar a produção ocorre em várias instâncias, desde as bancas examinadoras de dissertações e teses, até as apresentações que ocorrem nos congressos e agências financiadoras, passando pelo corpo editorial de periódicos.

Como a primeira instância de apresentação pública da produção científica muitas vezes ocorre em algum evento científico, passou a ser de interesse dos cientistas, da própria ciência e das agências financiadoras fazer a avaliação dos congressos, simpósios e eventos similares. É comum hoje que os 
promotores de eventos façam alguma avaliação solicitando aos participantes que manifestem sua opinião mediante a resposta a algum instrumento, em geral o questionário parece o mais usado. (GUZZO, WITTER, PFROMM NETO, ROSADO, WESCHESLER, 1995). Outra forma de avaliação que permite um julgamento mais objetivo é a análise cientométrica dos trabalhos apresentados no encontro.

Os eventos científicos cumprem várias funções no estatuto das ciências. Constituem excelente meio de comunicação entre os cientistas, dão visibilidade interna e externa ao seu trabalho, permitem uma perspectiva da produção gerada e conseqüentemente passa-se a dispor de evidências para aquilatar o desenvolvimento da área enfocada. Além disso, oferecem condições para que as pessoas se conheçam, estabeleçam relações produtivas de trabalho futuro, troquem informações, passem a integrar redes sociais de comunicação científica ou grupos de pesquisa. Vale acrescentar que, para muitos, a possibilidade de participar de eventos levando seus trabalhos é ao mesmo tempo uma variável motivacional forte a impulsionálos para a produção.

Nestas circunstâncias, não é de estranhar o crescimento ocorrido no século XX do investimento feito pelas agências financeiras em tais iniciativas. (FAPESP, 1973). O mesmo se pode dizer da explosão de ocorrência de tais encontros, visando ao atendimento de ampla gama de sociedades científicas, temáticas diversas, abrangendo grupos de pesquisadores de maior ou menor porte.

Dos eventos científicos tradicionalmente resulta a produção de anais que apresentam o que foi veiculado durante sua realização. Esta apresentação pode ser em termos de resumo, que é o mais freqüentemente usado, resumo expandido ou trabalho completo. Os anais constituem uma maneira eficiente de preservar a memória de instituições, sociedades científicas, eventos diversos. Acabam por se constituír em acervo para estudo da história, do desenvolvimento científico e tecnológico (MOTOYAMA, 2002). Além disso, durante o evento, representam um apoio substancial para o participante fazer seu plano pessoal de trabalhos que quer assistir.

Os anais hoje são impressos em papel ou em CD, sendo a última versão mais barata mas menos funcional no que diz respeito ao apoio ao participante, pois, para tanto, deverá ter um laptop ou contar com a disponibilidade de computadores em número suficiente nos hotéis e no próprio local do evento, o que nem sempre é viável. Entretanto, como documentação histórica cumpre sua função, embora possa não ter a mesma durabilidade do impresso em papel.

Dada a importância dos eventos científicos e conseqüentemente dos respectivos anais, há necessidade de fazer avaliações de diversos aspectos. Entre as avaliações possíveis está a da produção científica veiculada ao longo do acontecimento. Neste caso, a análise dos anais é uma forma de se poder, conduzindo um trabalho de metaciência, verificar o desenvolvimento da área, o nível dos trabalhos apresentados e obter base para uma perspectiva de desenvolvimento futuro.

Alguns exemplos de estudos de metaciência elaborados a partir de anais podem servir de referencial para o presente trabalho. $\mathrm{R}$ e i $\mathrm{n} \mathrm{h} \mathrm{o} \mathrm{ld}$ (2005) analisou a produção científica apresentada no $1^{\text {o }}$ Congresso Brasileiro de Stress, realizado em São Paulo, em 2003, quanto a tipo de estudo, temática, tipo de estresse, autoria, sujeitos e instrumentos usados na coleta. Encontrou indicativos positivos de desenvolvimento da área com produção mais marcante de psicologia escolar (31,6\%), de grande predomínio de pesquisa, só 5,4\% eram trabalhos teóricos, maior freqüência de autoria múltipla $(85,6 \%)$, sendo o Inventário de Sintomas de Stress de Lipp o instrumento mais utilizado.

Witter (1996) analisou comparativamente periódicos brasileiros, periódicos estrangeiros e anais de congressos de psicologia escolar enfocando a formação e a atuação do psicólogo na referida subárea da psicologia. Encontrou diferenças entre os suportes nacionais e estrangeiros predominando nos primeiros trabalhos de reflexão e nos segundos de pesquisa, sendo a atuação mais estudada do que a formação.

É neste quadro de referência que se estabeleceu a base para o trabalho aqui apresentado, tendo por preocupação básica a análise da produção veiculada em evento realizado na Inglaterra.

\section{Objetivos}

Constituíram-se como objetivos da presente metaciência analisar um evento científico na área de 
psicofisiologia quanto à autoria, tipo de trabalho, países e instituições participantes, temática e característica básica dos resumos.

\section{MÉTODO}

Evento pesquisado - Foram analisados os 85 trabalhos apresentados no British Psychophysiology Society Annual Meeting, realizado na Inglaterra, em Birmington, de 12 a 16 de setembro de 2005. Os anais foram publicados no Journal of Psychophysiology (2006), tendo-se acesso tanto em papel como via Internet, já que o periódico é disponibilizado nas duas versões. Para atingir os objetivos foram definidas categorias de análise para posterior tabulação.

\section{Autoria}

Por autoria, entenderam-se as pessoas que assinaram os textos. Não foi possível analisar o gênero dos produtores porque apenas a inicial dos pré-nomes constava dos anais. A análise focou a autoria quanto ao número de autores envolvidos, distinguindo-se autoria individual e autoria múltipla ou partilhada quando dois ou mais autores eram responsáveis pelo produto.

Exemplo de autoria individual é o trabalho de Bandettini (2006) sobre avaliação psicofisiológica funcional; como autoria múltipla, pode ser lembrada a apresentação de Burke e Barnes (2006) sobre a ativação cerebral durante controle preditivo de movimento do olho em seres humanos.

\section{Tipo de trabalho}

Foram estabelecidas duas categorias de trabalho: teórico e de pesquisa. No primeiro caso tratava-se de apresentação de uma reflexão teórica sobre o tema e no segundo na apresentação de relato de pesquisa, com dados diversos.

O trabalho teórico de Bandettini (2006) é um exemplo, pois trata-se de uma reflexão do autor sobre uma forma de avaliação que vem crescendo em termos de uso, com implicações para a prática e cuidados ao usá-la no futuro. Fawcett e Singh (2006) realizaram uma pesquisa para verificar a oscilação cortical dentro de faixas específicas de freqüência, investigando a evolução temporal ao movimento, visando três categorias de estímulos visuais apresentados a oito sujeitos. Concluíram que o tempo de reação inicial não pode ser usado conscientemente para se tomar decisão quanto à percepção do movimento.

\section{Título}

Estudou-se do título a característica extensão, considerada bom indicador de progresso, requerendo do responsável empenho para manter-se dentro de 12 vocábulos. Foram consideradas três possibilidades: títulos com até 9 , com 10 até 12 ou com mais de 13 vocábulos. Na primeira categoria se insere o trabalho Visual and auditory attention networks (CIRSTEA, BARRETT \& HALL, 2006) com cinco componentes. $\mathrm{Na}$ segunda pode ser mencionada a apresentação Calebrain: a hamonisation Project for multicentre structural and functional MRI, de Gountouna, McGonigle, Marshall, Hall, Cavanagh, Wyper, Waiter, Steele, Job, Moohead \& Lawrie (2006), contando 11 vocábulos. Na última categoria foram incluídos trabalhos como o de Van Horn (2006), com 13 vocábulos: Supporting hypothesis driven research through Discovery based Neuroscience: the MRI Data Center experience.

Outro aspecto estudado dos títulos foi a estrutura frasal usada pelo autor. O título deve ser uma oração declarativa afirmativa do trabalho. Não se espera que sejam usados títulos fantasia, nem orações interrogativas ou exclamativas. Nos anais estudados apareceram como título orações declarativas afirmativas e interrogativas. Exemplo do primeiro caso é o trabalho de Friston (2006) com o título Dynamic causal modelling of Brain Responses. No segundo caso, citam-se Begliomini, Smith, Wall e Castiello (2006), com Precision versus whole-hand visually guided grasping: is AIP always necessary?

\section{Países participantes}

Foi feito um mapeamento dos países participantes tendo por base a vinculação do autor a alguma instituição. Nestas circunstâncias, o trabalho de Cloutier, Turk e Macrae (2006) foi computado para os Estados Unidos, já que os três autores eram do Dartmouth College, de Hanover, do referido país e mesma instituição. Já para comunicação feita por Dyson, Alain e He (2006), foram contadas contribuições para a Inglaterra, pois o primeiro autor é da University of Sussex, outra para o Canadá, posto que Alain está vinculado a The Rotman Research Institute, instituição canadense. Outro registro foi feito para o mesmo país, já que He é da University of Toronto. 


\section{Instituições participantes}

Tabularam-se as instituições participantes tendo por referencial seu nome principal, isto é, não foram consideradas subdivisões como departamento, serviços, grupos de pesquisa ou trabalho. Os exemplos do item anterior são válidos também para a presente análise.

\section{Temática}

Cada trabalho pode enfocar um ou mais temas, tendo se registrado cada um deles. Como houve grande dispersão, posteriormente foram aglutinados em categorias por conteúdo. $\mathrm{O}$ primeiro registro resultou em 147 assuntos.

Estrutura e funcionamento do cérebro - inclui trabalhos enfocando a organização estrutural e o funcionamento do cérebro, como no estudo de David, Cohen, Bewernick, Newen, Fink, Shah e Vogeleu (2006) sobre os mecanismos neurais envolvidos na identificação que a pessoa faz de suas próprias ações.

Órgãos dos sentidos - trata-se de trabalhos que enfocam atividades dos órgãos do sentido visual ou auditiva. É exemplo o trabalho de Field, Wilkie e Wann (2006) sobre a percepção de informação visual.

Avaliação neurológica - compreende os trabalhos que privilegiam instrumentos, recursos e a avaliação propriamente dita geral ou parcial, do contexto neurológico. Um exemplo é o texto com o título MEG, de Hillebrand (2006), referente a instrumento usado para avaliação neural que fornece uma visão do que ocorre no cérebro.

Atividade física - composta por trabalhos que incluíram aspectos da atividade física entre as variáveis focadas. Exemplo é o trabalho de Hughes, Velmans e de Fockert (2006) sobre a possibilidade de inibição inconsciente da resposta motora.

Cognição - a categoria incluiu estudos sobre memória, atenção, cognição e linguagem, como na produção de Humphreys (2006) sobre diferenças inter e intrapessoas sobre o funcionamento cognitivo.

Problemas biopsicológicos - trata-se de relatos sobre problemas de saúde físicos (cardiopatia) ou psicológicos (autismo, psicose), envolvendo ou não remédios, como na apresentação de Bullmore (2006) sobre distúrbios psiquiátricos estudados via imagem estrutural do cérebro.
Aspectos psicológicos não patológicos - são textos sobre aspectos afetivos emocionais sem envolver patologias. Um exemplo é o estudo de Ischai (2006) sobre avaliação de percepção de faces enfocando o efeito das variáveis memória, emoção e gênero.

Outras - nesta categoria foram incluídos temas que aparecem com freqüência muito baixa e que não se enquadravam nas demais categorias, como no caso do gênero. Exemplo o trabalho de Lau, Butler e Senior (2006) sobre os efeitos do ciclo menstrual nas negociações de trabalho.

índice de concordância $[\overline{\overline{I C=A x 100}} \overline{A+D}]$ $\boldsymbol{A}+\boldsymbol{D} \quad$ resultou em $87 \%$ quando dois juízes fizeram a categorização dos temas, sendo possível de se considerar fidedigno o resultado obtido.

\section{Estrutura geral do resumo}

Como é esperado que resumos sejam uma peça discursiva de um parágrafo, analisou-se este aspecto foi observado nos trabalhos apresentados no evento. Duas possibilidades foram previstas: com e sem parágrafo. No primeiro caso, citam-se Smith e Senior (2006) que fizeram um parágrafo novo para relatar os resultados de sua pesquisa. No segundo, tem-se o trabalho de Wood (2006), sem parágrafo.

\section{RESULTADOS E DISCUSSÃO}

Do evento participaram 23 autores que apresentaram trabalhos individuais e 62 trabalhos em autoria múltipla. Para verificar se essa diferença era significante, aplicouse $\mathrm{o} \div 2\left(\mathrm{H}_{\mathrm{o}}: \div 2\right.$ zero; $\mathrm{H}_{\mathrm{a}}: \div 2$ “" zero) sendo pd" $0,05, \div \div_{\mathrm{c}}=$ 3,84. Foi obtido $\div 2{ }_{0}=17,99$ portanto rejeitou-se $\stackrel{c}{H}_{\text {o, }}$ sendo estatisticamente significante a maior presença de trabalhos em autoria múltipla, o que indica cientometricamente que se está diante de um indicador de bom desenvolvimento científico. Estes resultados são consoantes aos encontrados por Reinhold (2005) e Witter (1996).

Em relação aos trabalhos apresentados, apenas dois foram teóricos e 83 relataram pesquisas (98\%); pelo fato do número de pesquisa ser tão elevado, na última categoria dispensa-se o cálculo. Sua relevância indica que se trata de um evento de alto nível, por dominar os trabalhos de pesquisa, conforme os critérios das normas vigentes, como é mencionado em Comunicação $\mathscr{E}$ Produção Científica, organizado por Poblacion, Witter e Silva (2006. Os dados obtidos são similares ao registrado por Reinhold (2005). 
TABELA 1

Análise da extensão do título

\begin{tabular}{|llc|}
\hline Variação & F & $\%$ \\
\hline Até 9 & 28 & 32,94 \\
\hline 10 a 12 & 25 & 29,41 \\
\hline mais de 13 & 32 & 37,65 \\
\hline Total & 85 & 100 \\
\hline
\end{tabular}

Os resultados da análise de título encontram-se na tabela 1 , sendo que a maior freqüência das categorias foi para 13 ou mais vocábulos, indicativos de que os profissionais da área têm de ter maior cuidado com a elaboração dos títulos enviados para o evento. Também é indicativo que os organizadores do evento ou membros da comissão científica não estiverem atentos a estes detalhes, para definir critérios mais claros e específicos para apresentação dos trabalhos. Foi feita a análise estatística recorrendo-se ao teste que $\div$ para $\mathrm{H}_{\mathrm{o}}: \div^{2}=$ zero; $\mathrm{H}: \div \div^{2}=$ zero) sendo pde 0,05 , n.g.l. $=2 \mathrm{e} \div{ }^{2}=$ $5,99, \mathrm{o} \div 2=0,87$, permitindo concluir que não houve diferença entre as três categorias. Vale dizer que os títulos poderiam ser refinados.

No que concerne à estrutura frasal escolhida pelo autor, foram obtidos 76 títulos na forma declarativa afirmativa $(88,2 \%)$ e nove interrogativas $(11,8 \%)$, sendo dispensável o cálculo em função da forte concentração na categoria esperada. Novamente se está diante de bom indicador de qualidade favorável ao evento.

No que se refere à participação dos vários países, os resultados são apresentados na tabela 2, verificando-se

TABELA 2

\section{Presença dos Países no Evento}

\begin{tabular}{|l|l|r|}
\hline País & F & $\%$ \\
\hline Inglaterra & 67 & 55,4 \\
\hline USA & 13 & 10,7 \\
\hline Alemanha & 13 & 10,7 \\
\hline Itália & 5 & 4,1 \\
\hline Escócia & 4 & 3,3 \\
\hline Taiwan & 3 & 2,5 \\
\hline Suíssa & 3 & 2,5 \\
\hline Canadá & 2 & 1,7 \\
\hline Polônia & 2 & 1,7 \\
\hline Austrália & 2 & 1,7 \\
\hline Hong-Kong & 2 & 1,7 \\
\hline Cuba & 1 & 0,8 \\
\hline Holanda & 1 & 0,8 \\
\hline Nova Zelândia & 1 & 0,8 \\
\hline Brasil & 1 & 0,8 \\
\hline Eslovência & 1 & 0,8 \\
\hline Total & 121 & 98 \\
\hline & & \\
\hline
\end{tabular}

TABELA 3

Instituições Participantes

\begin{tabular}{|l|l|l|}
\hline Instituições & F & $\%$ \\
\hline Aston University & 13 & 15,9 \\
\hline University of London & 6 & 7,3 \\
\hline Welcome Dep. Of Imaging Neuroscience & 4 & 4,1 \\
\hline Institute of Psychiatry of London & 3 & 3,7 \\
\hline MRC Institute of Hearing Research & 3 & 3,7 \\
\hline University of Cambridge & 3 & 3,7 \\
\hline University of Manchester & 3 & 3,7 \\
\hline Outras (freq. 2 ou 1) & 47 & 57,3 \\
\hline Total & 82 & 100,2 \\
\hline
\end{tabular}

que, como era de se esperar pelo local do evento, maior participação de trabalhos foi da Inglaterra $(55,4 \%)$.

Em seguida, empatados aparecem Estados Unidos e Alemanha com 10,7\% . Seguem Itália (4,1\%), Escócia $(3,3 \%)$ e os demais com ocorrência ainda mais baixa. Vale fazer notar que a presença européia é maior sendo raros os trabalhos de outros continentes, o que pode ser explicado pela proximidade geográfica e alto custo para participação no evento para pessoas de outros continentes.

Considerando que a existência de grupos e de linhas de pesquisa em instituições pode alavancar o conhecimento de forma mais sistemática e regular em termos de ritmo de produção, foi estudada a contribuição em termos de vinculação institucional. Os resultados aparecem na tabela 3, sendo de se destacar que houve dispersão, com poucos pontos de concentração.

Ao todo se fizeram representar 82 instituições, sendo a maioria universidades. Dada a grande dispersão, na tabela 3 foram inseridas apenas as instituições com maior presença. Em primeiro lugar ficou a Aston University (15,9\%), seguida da University of London (7,3\%), ficando as demais com participação muito pequena.

No que concerne aos temas, também foi registrada uma ampla variação $(\mathrm{N}=147)$, mas foi possível refinar a análise definindo categorias para reorganizar os dados obtidos. Os resultados aparecem na tabela 4 a seguir.

Predominam os trabalhos sobre estrutura e funcionamento do cérebro $(25,2 \%)$, órgãos do sentido $(18,4 \%)$ e cognição $(12,9 \%)$ e avaliação neurológica $(10,2 \%)$. Para efeito de verificar se a distribuição era homogênea foi feito o teste de $\div 2\left(\mathrm{H}_{\mathrm{o}}\right.$ : $\div^{2}=$ zero; $\mathrm{H}_{\mathrm{a}}: \div^{2}$ “"zero, pd" 0,05, n.g.1.=7, excluindo-se a categoria outros). Foi obtido $\div$ 。 
TABELA 4

Temática enfocada nos trabalhos

\begin{tabular}{|l|c|c|}
\hline Tema & F & $\%$ \\
\hline Estrutura e Funcionamento do cérebro & 37 & 25,2 \\
\hline Órgãos do Sentido & 27 & 18,4 \\
\hline Avaliação Neurológica & 15 & 10,2 \\
\hline Atividade Física & 11 & 7,5 \\
\hline Cognição & 14 & 9,5 \\
\hline Problemas biopsicológicos & 13 & 8,8 \\
\hline Aspectos psicológicos não patológicos & 9 & 6,1 \\
\hline Cognição & 19 & 12,9 \\
\hline Outros & 2 & 1,4 \\
\hline Total & 47 & 100 \\
\hline
\end{tabular}

$=31,91$ que comparado ao valor crítico de 14,07 permite concluir que a concentração em estrutura e funcionamento cerebral $(19,67)$ responde pela significância encontrada já que as demais categorias ficaram dentro do estatisticamente esperado.

A análise da estrutura dos resumos mostrou que apenas seis deles apresentaram parágrafo (7,06\%) estando a maioria $(92,9 \%)$ dentro do parâmetro esperado, isto é, sem parágrafo (DOMINGOS, 1999). A diferença torna desnecessário qualquer cálculo e permite dizer que os trabalhos tendem ao padrão discursivo de resumo cientificamente recomendado.

\section{CONCLUSÕES}

A análise da produção científica veiculada ao longo do encontro de psicofisiologia aqui enfocado permite concluir que foi um evento de alto nível, conforme demonstram a análise de autoria, tipo de trabalho, estrutura do resumo e mesmo, em parte, pela formulação do título.

A maior participação do país que sediou o evento era esperada, o mesmo podendo-se dizer dos países europeus em função da situação geográfica e facilidade financeira para comparecimento. Dentre as instituições participantes destacam-se também, pelas razões já expostas, as da Inglaterra e países mais próximos.

A temática do evento ficou criteriosamente dentro da área, sendo que das várias possibilidades, significativamente houve preferência por apresentar informações sobre a estrutura e funcionamento do cérebro, assunto básico para todas as demais categorias. Isto indica capacidade seletiva e hierarquizada da temática o que também é positivo quanto à qualidade do evento e indício de bom desenvolvimento científico.
No que concerne aos títulos, autores e comissão científica, poderiam ter tido um pouco mais de cuidado, mas, as diferenças não sendo significantes, não cabe mais do que um alerta para melhorar este aspecto nos próximos eventos.

Em síntese, foi um evento produtivo, com grande adesão aos indicadores de produtividade científica.

Artigo submetido em 22/08/2007 e aceito em 14/04/2008.

\section{REFERÊNCIAS}

ALTMAN, C. A pesquisas lingüística no Brasil (1968/1988). São Paulo: Humanitas, 2005.

BANDETTINI, P. Functional MRI in perspective. Journal of Psychophysiology, v. 20, n. 4, p. 315-336, 2006. Abstracts of British Psychophysiology Society Annual Meeting.

BERTERO, C. O.; CALDAS, M. P.; WOOD JR., T. Produção científica em administração no Brasil: o estado da arte. São Paulo: Atlas, 2005.

BULLMORE, F. Imaging causes and cures of psychiatric disorders. Journal of Psychophysiology, v. 20, n. 4, p. 315-336, 2006. Abstracts of British Psychophysiology Society Annual Meeting.

BURKE, M.; Barnes, G. Brain activation during predictive control of eye movements in humans. Journal of Psychophysiology, v. 20, n. 4, p. 315-336, 2006. Abstracts of British Psychophysiology Society Annual Meeting.

CIRTEA, S.; BARRETT, D.; HALL, D. Visual and auditory attention networds. Journal of Psychophysiology, v. 20, n. 4, p. 315-336, 2006. Abstracts of British Psychophysiology Society Annual Meeting.

CLOUTIER, J.; TURK, D.; MACRAE, N. The extraction of gender and Gaze Information: an investigation of their relationship and neural substrate. Journal of Psychophysiology, v. 20, n. 4, p. 315-336, 2006. Abstracts of British Psychophysiology Society Annual Meeting.

DAVID, N. Cohen et al. Neural mechanisms involved in the identification of one's own action. Journal of Psychophysiology, v. 20, n. 4, p. 315-336, 2006. Abstracts of British Psychophysiology Society Annual Meeting. 
DOMINGOS, N. A. M. Produção científica: análise de dissertações e teses em psicologia (1992/1996). 1999. Tese (Doutorado em Pscologia) - PUC-Campinas, 1999.

DYSON, B.; ALAIN, C.; He, Y. Exogenous and endogenous contributions to acoustic perceptual invariance. Journal of Psychophysiology, v. 20, n. 4, p. 315 336, 2006. Abstracts of British Psychophysiology Society Annual Meeting.

FAWEETL, I.; SINGH, K. Early and late correlates of implied motion perception in human motion area V5/ MT. Journal of Psychophysiology, v. 20, n. 4, p. 315 336, 2006. Abstracts of British Psychophysiology Society Annual Meeting.

FIELD, D.; WILDIE, R.; WANN, J. Functional localization of visual dorsal stream areas involved insteering a palk based on visual information. Journal of Psychophysiology, v. 20, n. 4, p. 315-336, 2006. Abstracts of British Psychophysiology Society Annual Meeting.

FUNDAÇÃO DE AMPARO À PESQUISA DO ESTADO DE SÃO PAULO - FAPESP. Pesquisa $\mathcal{E}^{2}$ desenvolvimento. São Paulo, 1973.

GOUNTOUNA, V. E. et al. Abstracts of British Psychophysiology Society Annual Meeting. Journal of Psychophysiology, v. 20, n. 4, p. 315-336, 2006.

GUZZO, R. S. L. et al. O future da criança na escola, familia e sociedade: the child's future in school, family and society. Campinas: Átomo, 1995.

HILLEBRAND, A. MEG. Journal of Psychophysiology, v. 20, n. 4, p. 315-336, 2006. Abstracts of British Psychophysiology Society Annual Meeting.

HUGHES, G.; VELMANS, M.; FOCKERT, J. de. Can motor responses be inhibited inconsciously?. Journal of Psychophysiology, v. 20, n. 4, p. 315-336, 2006. Abstracts of British Psychophysiology Society Annual Meeting.

HUMPHREYS, G.W. Inter and intraindividual differences in cognitive functioning: single case approaches in cognitive neuroscience. Journal of Psychophysiology, v. 20, n. 4, p. 315 336, 2006. Abstracts of British Psychophysiology Society Annual Meeting.
ISCHAI, A. FMRI studies of face perception: effects of memory, emotion and sexual preference. Journal of Psychophysiology, v. 20, n. 4, p. 315-336, 2006. Abstracts of British Psychophysiology Society Annual Meeting.

LAU, A.; BUTLER, M.; SENIOR, C. The menstrual cycle affects job negotiation. Journal of Psychophysiology, v. 20, n. 4, p. 315-336, 2006. Abstracts of British Psychophysiology Society Annual Meeting.

MOTOYAMA, S. (Org.). 50 Anos do CNPq: contados pelos seus presidentes. São Paulo: FAPESP, 2002.

POBLACIÓN, D.; WITTER, G. P.; SILVA, J. F. M. da. Comunicação \% produção científica: contexto, indicadores e avaliação. São Paulo: Angellara, 2005.

REINHOLD, H. H. Análise da produção científica de um Congresso Brasileiro de Estresse. In: WITTER, G. P. (Org.). Metaciência e psicologia. Campinas: Alínea, 2005. Cap. 6, p. 111-136.

SMITH, D.; SENIOR, C. Individual differences and judgments of male beauty across menstrual cycle. Journal of Psychophysiology, v. 20, n. 4, p. 315-336, 2006. Abstracts of British Psychophysiology Society Annual Meeting.

VANHORM, J. D. Supporting hypothesis driven research through discovery based neuroscience: the MRI Data Center experience. Journal of Psychophysiology, v. 20, n. 4, p. 315-336, 2006. Abstracts of British Psychophysiology Society Annual Meeting.

WOOD, L. MRSA: a new possibilities in streamlining bock design FMRJ: analysis of AFNI. Journal of Psychophysiology, v. 20, n. 4, p. 315-336, 2006. Abstracts of British Psychophysiology Society Annual Meeting.

WITTER, G. P. A produção científica no universo das ciências humanas. In: ENCONTRO DE PESQUISADORES NA UNIVERSIDADE SÃO FRANCISCO, 2., 1994, Bragança Paulista. Anais... Bragança Paulista: USF, 1994. Falta página.

WITTER, C. Psicologia escolar: produção científica, formação e atuação (1990-1994). 1996. Tese (Doutorado em Psicologia) - Instituto de Psicologia, Universidade de São Paulo, São Paulo, 1996. 